\title{
LA TROPICALIZACIÓN DEL PRINCIPIO DE PROPORCIONALIDAD: \\ LA EXPERIENCIA DE COLOMBIA Y MÉXICO EN EL ÁMBITO DE IGUALDAD
}

LUISA CONESA LABASTIDA 


\section{SUMARIO}

I. INTRODUCCIÓN. II. ¿¿QUÉ ES EL PRINCIPIO DE PROPORCIONALIDAD? III. APLICACIÓN DEL PRINCIPIO DE PROPORCIONALIDAD AL PRINCIPIO DE IGUALDAD EN ALEMANIA. IV. VARIABLE INTENSIDAD EN LOS TESTS NORTEAMERICANOS EN MATERIA DE IGUALDAD. V. EL CASO COLOMBIANO. 1. PROPORCIONALIDAD COMO PARÁMETRO DE IGUALDAD. 2. TEST DE RAZONABILIDAD. 3. TRES TIPOS DE TESTS. 4. TEST(S) INTEGRADO. V. EL CASO MEXICANO. 1. SURGIMIENTO DEL «TEST DE RAZONABILIDAD». 2. MODULACIÓN EN EL ESCRUTINIO MEXICANO. 3. APLICACIÓN FINALIZADA DEL TEST MEXICANO. VI. CONCLUSIÓN: LA TROPICALIZACIÓN DEL PRINCIPIO DE PROPORCIONALIDAD. 


\title{
LA TROPICALIZACIÓN DEL PRINCIPIO DE PROPORCIONALIDAD: LA EXPERIENCIA DE COLOMBIA Y MÉXICO EN EL ÁMBITO DE IGUALDAD
}

\author{
POR \\ LUISA CONESA LABASTIDA \\ Instituto Tecnológico Autónomo de México
}

\section{INTRODUCCIÓN}

Desde que el modelo de Estado Constitucional remplazó el paradigma del Estado Legislativo - trayendo como resultado que la Constitución ocupe la cúspide de la pirámide normativa - la cuestión de cómo interpretarla ha ocupado las mentes tanto de los jueces constitucionales como de estudiosos del derecho. La problemática es compartida tanto por los sistemas de origen europeo como por el modelo norteamericano de judicial review, pues siempre que haya que descifrar el significado del texto constitucional habrá debates en torno a la mejor manera de llevar a cabo esta tarea.

Si bien es cierto la problemática es similar, las herramientas utilizadas para solucionarla han variado en ambos lados del Atlántico. En el campo de adjudicación de derechos la Corte Suprema Americana y la tradición europea han desarrollado técnicas diferenciadas: aquélla ha emitido un gran acervo de jurisprudencia que establece una variedad de tests de intensidad gradual, desde el más débil estándar de rational basis review hasta el casi insuperable strict scrutiny, 
mientras que ésta ha tomado un camino diferente — particularmente en los últimos veinte años - identificada como el principio de proporcionalidad.

El uso de dicho método comenzó esencialmente con los avances jurisprudenciales del Tribunal Constitucional Alemán, el cual desarrolló un mecanismo innovador que gradualmente se ha convertido en lenguaje común para la argumentación constitucional en diversas latitudes. En Europa la jurisprudencia alemana ha sido emulada - en mayor o menor medida - por los tribunales constitucionales de Francia, Italia, Portugal, Austria, Hungría, Checoslovaquia, Eslovaquia, Estonia, España y Suiza. ${ }^{1}$

En el ámbito del derecho internacional el principio es visible en la jurisprudencia del Tribunal Europeo de Derechos Humanos y en la del Tribunal de Justicia de la Unión Europea. Tal es su expansión que incluso ha trascendido el nivel jurisprudencial para ser positivizado en el texto de la Carta Europea de Derechos Fundamentales (artículo 52.1), la Constitución europea (artículos II.109.3 y II.112.1) la Constitución de Berna de 1993 (numeral 28.3) y la Constitución Portuguesa (artículo 18.2). ${ }^{2}$

Aunque en menor medida que en Europa, América Latina no se ha quedado atrás en cuanto al principio de proporcionalidad se refiere. Toda vez que los países de esta región tradicionalmente tienden a ser más receptivos de las modas norteamericanas en oposición a las europeas, este trabajo trata de analizar cómo Colombia y México han importado el referido principio, preguntándose si han adoptado el modelo tradicional alemán, si han retenido la acostumbrada influencia norteamericana y si han aportado nuevos elementos que, incluso, pudieran ser mejoras para los modelos que les dieron origen.

Se les toma como casos paradigmáticos debido a que brindan la oportunidad de contrastar una corte joven con una institución con más de un siglo de existencia, cada una con su propio estilo argumentativo. La Corte Constitucional de Colombia es conocida como una de las más progresistas en América Latina - y probamente a nivel internacional- a pesar de su reciente creación en 1991. En contraste, la Suprema Corte de Justicia de la Nación mexicana es mejor descrita como una organización tradicional y madura, funcionando incluso antes de la promulgación de la Constitución de 1917 vigente en la actualidad. ${ }^{3}$

1 Toda vez que el juicio de proporcionalidad es un producto Europa en general y de Alemania en particular, en este artículo se utilizan indistintamente los términos «test europeo y «test alemán».

2 Cfr. Bernal Pulido, Carlos, El Principio de proporcionalidad y los derechos fundamentales, Centro de Estudios Constitucionales, Madrid, 2007, pp. 50, 51, 55, 56.

${ }_{3}$ Es importante mencionar que si bien es cierto la Corte Mexicana ha dictado sentencias desde mucho antes de la entrada en vigor de la Constitución de 1917, la jurisprudencia que se con- 
El presente análisis es guiado por las siguientes preguntas: ¿qué es el principio de proporcionalidad? ¿Cuál es la relación entre los principios de proporcionalidad e igualdad? ¿Cómo se han utilizado por las cortes colombiana y mexicana y cuáles son sus influencias? ¿Se hacen referencias expresas al derecho comparado para justificar la inclusión del test? ¿Han importado el tradicional test Europeo? ¿'Se ha retenido la influencia norteamericana?

La hipótesis del presente artículo es que, en el proceso de adaptación del principio de proporcionalidad a las circunstancias de sus propias jurisdicciones, ambas cortes han tropicalizado el modelo. Este término no es en absoluto utilizado en afán peyorativo sino como una manera de identificar y explicar una parte del proceso globalizador visible en la dinámica de las diversas cortes constitucionales, en virtud del cual las doctrinas son importadas y adaptadas a las necesidades de cada país. Idealmente el producto final aporta los beneficios disfrutados en otras jurisdicciones sin dejar de lado las particularidades del Estado receptor.

Por lo que al principio de proporcionalidad se refiere, se sostiene que se la tropicalización es visible en el entramado jurisprudencial que combina características propias de las jurisdicciones colombiana y mexicana, respectivamente, más la suma de elementos del test alemán y rasgos de la jurisprudencia norteamericana. ${ }^{4}$

\section{II. ¿QUÉ ES EL PRINCIPIO DE PROPORCIONALIDAD?}

Este canon nace como una herramienta interpretativa para una nueva generación de constitucionalismo que ve al texto constitucional no únicamente

sidera válida es la emitida a partir de este año, denominada Quinta Época — la emitida con anterioridad sólo interesa para fines históricos-. Otra nota relevante es que el Poder Judicial de la Federación Mexicana atravesó una completa restructuración a partir de la reforma del texto constitucional de 1994, la cual efectivamente trasformó a la Suprema Corte en una Corte Constitucional con facultades de control abstracto, concreto y protección de derechos fundamentales mediante demandas presentadas directamente por los ciudadanos — se trata de la acción de inconstitucionalidad, controversia constitucional y juicio de amparo, respectivamente-.

4 Toda vez que este trabajo se centra más en el desarrollo jurisprudencial latinoamericano que en la conceptualización del principio de proporcionalidad y los tests norteamericanos, el espacio que se les dedica es menor y con el único fin de esbozar a grandes rasgos sus características esenciales para después identificarlas en las propias jurisprudencias. Para profundizar en cualquiera de ellos se recomiendan, entre otras, la obras de Carlos Bernal Pulido (El Principio de proporcionalidad y los derechos fundamentales, Centro de Estudios Constitucionales, Madrid, 2007) y Richard Fallon (Implementing The Constitution, Harvard University Press, Massachussets, 2001). 
como un conjunto de reglas sino como un universo más vasto que contiene también principios y valores y que, por ende, requiere de una aproximación ad hoc.

Se trata de uno de los conceptos surgidos a partir del denominado neoconstitucionalismo. Esta corriente relativamente reciente sostiene que el moderno Estado Constitucional presenta novedades teóricas y prácticas tanto en el propio texto de sus constituciones — adoptadas después de la segunda guerra mundial y particularmente a partir de los años setenta - como en las prácticas jurisprudenciales y técnicas interpretativas que surgen a partir de aquéllas. ${ }^{5}$

Los problemas que llaman a la utilización de la proporcionalidad no son los — tomando la terminología de Dworkin - casos fáciles en los cuales hay un choque entre reglas, sino los aquéllos difíciles en los que existe un conflicto que involucra principios y valores y que exige — dada su inconmesurabilidaduna aproximación diferenciada.

La ponderación como característica esencial del principio de proporcionalidad posee un carácter particularizado en relación al uso argumentativo tradicional del término, el cual que se refiere a una técnica general aplicable a todos aquellos supuestos en los que existan valores o intereses en conflicto. En el primer caso se trata de ponderar un interés público con un interés privado, mientras que en el segundo se enfrentan, de manera horizontal, dos intereses privados. ${ }^{6}$

Robert Alexy explica que la naturaleza de los principios en general y del principio de proporcionalidad en particular, son dos cuestiones que van de la mano:

Ya se ha insinuado que entre la teoría de los principios y la máxima de proporcionalidad existe una conexión. Esta conexión no puede ser más estrecha: el carácter de principio implica la máxima de proporcionalidad, y ésta implica aquélla. Que el carácter de principio implica la máxima de la proporcionalidad significa que la máxima de la proporcionalidad, con sus tres máximas parciales de la adecuación, necesidad (postulado del medio más benigno) y de la proporcionalidad en sentido estricto (el postulado de la ponderación propiamente dicho) se infiere lógicamente del carácter de principio, es decir, es deducible de él. El Tribunal Constitucional ha dicho, en una formulación algo oscura, que la máxima de la proporcionalidad resulta «en el fondo ya de la propia esencia de los derechos fundamentales». ${ }^{7}$

5 Acerca del neoconstitucionalismo $c f r$. Carbonell, Miguel, «El Neoconstitucionalismo en su Laberinto» en Teoría del neoconstitucionalismo, Trotta, Madrid, 2007.

${ }^{6}$ Cfr. Sarmiento Ramírez-Escudero, Daniel, El control de la proporcionalidad de la actividad administrativa, Tirant lo Blanch, Valencia, 2004, p.392.

7 Alexy, Robert, Teoría de los derechos fundamentales, traducción de Ernesto Garzón Valdez, Centro de Estudios Constitucionales, Madrid, 1993, pp. 111,112. 
En cuanto al origen de su utilización, la doctrina señala que fue en Europa a finales de la década de los años sesenta cuando se recurre a este principio en la doctrina constitucional como parámetro para juzgar las actividades de los poderes públicos que intervienen en los derechos fundamentales. ${ }^{8}$ Sánchez González explica que el Tribunal Constitución Alemán reconoció en 1968 que — derivado de la cláusula del Estado de Derecho- la prohibición de exceso (Übermassverbot) y el principio de proporcionalidad (Verhältnismäs-sigkeitsprinzip) eran reglas aplicables a las actividades estatales que conlleven una restricción a las libertades de los ciudadanos.

En el subsiguiente desarrollo terminológico la prohibición de exceso y la proporcionalidad en sentido amplio fueron tratadas como equivalentes, mientras que la proporcionalidad en sentido estricto se apuntaló como el requisito de ponderación (Abwägung). Esta última se introdujo en el famoso caso Luth, en el cual la Corte argumentó que la Constitución debe comprenderse como un todo dinámico que conlleva una escala objetiva de valores, por lo cual el intérprete está obligado a realizar una ponderación en el contexto del caso particular. ${ }^{9}$

En términos generales puede afirmarse que el test se encuentra encaminado a dilucidar si una determinada injerencia estatal en las libertades individuales es permisible en términos constitucionales, realizando una ponderación entre los principios que compiten entre sí.

En la aplicación del principio de proporcionalidad al caso concreto, el Tribunal Constitucional alemán utiliza un test tripartito - tres niveles desarrollados de manera escalonada, de tal suerte que para continuar al segundo paso es necesario primero haber hecho un pronunciamiento sobre el primero y así sucesivamente-, que evalúa la constitucionalidad de la injerencia estatal en las libertades individuales. Se desarrolla de la siguiente manera:

a) Idoneidad o Adecuación (Geeignetheit): Toda vez que la medida legislativa o acción estatal debe ser coherente con el fin legítimo que fue diseñada para alcanzar, pasará este nivel del test si es adecuada —en términos de una causalidad lógica entre medios y fines- para la consecución del fin

${ }^{8}$ Debe resaltarse que el uso del principio de proporcionalidad como prohibición de exceso de los poderes públicos se origina en el Derecho Administrativo prusiano en el siglo XIX y posteriormente se incorpora como canon constitucional cfr. Giménez Glück, David, Juicio de igualdad y tribunal constitucional, Bosch, Barcelona, 2004, p. 92. La doctrina señala que la génesis concreta se encuentra en el caso de las farmacias resuelto por el Tribunal Constitucional Alemán en 1952, cfr. Bernal Pulido, Carlos, Op. cit., pp. 54, 55.

9 Sánchez González, Santiago, «De la imponderable ponderación y otras artes del Tribunal Constitucional», en Teoría y Realidad Constitucional, número 12, Madrid, 2003, pp. 9, 10. 
b) Necesidad (Erforderlichkeit o Notwendigkeit): «Toda medida de intervención en los derechos fundamentales deber ser la más benigna con el derecho intervenido, entre todas aquéllas que revisten por lo menos la misma idoneidad para contribuir a alcanzar el mismo objetivo». ${ }^{10}$ Dicho de otro modo, la cuestión en este punto es si el fin puede ser alcanzado con idéntica eficacia utilizando otra medida menos lesiva para el individuo.

c) Proporcionalidad en sentido estricto o ponderación (Proportionalität or Abwägung). El ultimo paso consiste en preguntarse si a pesar de que la medida está suficientemente acotada por los primeros dos estándares falla al infringir en el derecho más de lo debido, esto es, si la restricción al derecho desplazado es excesiva. El mandato consiste en que «el acto o disposición general no restringirá los intereses del particular de forma desproporcionada, en relación a los beneficios generales que se pretende alcanzar». ${ }^{11}$ Este último paso es el núcleo del test alemán y que podría encuadrarse dentro de lo que Dworkin caracterizaría como tomarse los derechos en serio. ${ }^{12}$

En el ámbito doctrinal Alexy señala que este tercer sub-principio se identifica con su propia Ley de Ponderación, la cual prescribe: cuanto mayor es el grado de la no satisfacción o de afectación de un principio, tanto mayor tiene que ser la importancia de la satisfacción del otro. ${ }^{13} \mathrm{El}$ autor explica que mientras los principios son mandatos de optimización en relación con las posibilidades jurídicas y fácticas existentes, los tres sub-principios en cuestión no son principios en sentido estricto sino reglas, dado que su insatisfacción trae como resultado la irregularidad de la norma de cara al sistema.

En este orden de ideas, mientras el principio de proporcionalidad en sentido estricto se sigue del hecho de que los principios son mandatos de optimización respecto de lo que es jurídicamente posible, los de necesidad y adecuación se siguen de lo que es fácticamente posible. ${ }^{14}$

\section{APLICACIÓN DEL PRINCIPIO DE PROPORCIONALIDAD AL PRINCIPIO DE IGUALDAD EN ALEMANIA}

Como atinadamente señala Giménez Glück, «el principio de proporcionalidad es una figura jurídica que excede del ámbito de la igualdad, que no es más

\footnotetext{
${ }^{10}$ Bernal Pulido, Carlos, Op. cit., p. 42.

${ }_{11}$ Sarmiento Ramírez-Escudero, Daniel, Op. cit., p.29.

12 Cfr. Dworkin, Ronald, Taking Rights Seriously, Duckworth, Londres, 1978.

13 Cfr. Alexy, Op. cit., p. 161.

${ }^{14}$ Ibid., p. 112.
} 
que una de las materias donde dicho principio se aplica». Se trata de un principio propio de la doctrina general de los derechos fundamentales, que se dirige al acotamiento de la actividad estatal limitadora de derechos sea por medio de actuaciones administrativas o mediante normas procedentes del legislador. ${ }^{15}$

En sus orígenes el principio de proporcionalidad no era utilizado como canon aplicable a las demandas presentadas en torno al principio de igualdad, el cual - hasta 1980 - formalmente era juzgado por el Tribunal Constitucional Alemán bajo el estándar más laxo de prohibición a la arbitrariedad. Con base en éste existirá una vulneración en el derecho sólo «en cuanto no sea posible encontrar un motivo razonable derivado de la naturaleza de la cosa o de cualquier otro motivo objetivamente lógico, es decir, cuando la determinación debe calificarse de arbitraria». ${ }^{16}$

Dicho estándar — que, como se verá enseguida, guarda similitudes con el rational basis review norteamericano- fue modificado por el Tribunal Alemán en sentencia de 7 de octubre de 1980, debido a que su deferencia legislativa contrastaba con la protección más amplia otorgada a los derechos de libertad al amparo del principio de proporcionalidad. Villacorta Mancebo explica que bajo esta «nueva fórmula» existirá una violación a la igualdad «si un grupo de afectados por la norma recibe por comparación a otros grupos un tratamiento desigual, pese a que entre ambos colectivos no existan diferencias de tal naturaleza e importancia que pudieran justificar la diferencia de trato (...) en fin, la sentencia parece poner asimismo el acento en el aspecto de la proporcionalidad de la igualdad». ${ }^{17}$

Ahora bien, a partir de este momento la doctrina critica la falta de uniformidad en cuanto a la relación entre proporcionalidad e igualdad en la jurisprudencia alemana. Sobre esta cuestión se alzan voces que consideran que el paso de arbitrariedad a proporcionalidad fue meramente formal pero que en realidad «la Segunda Sala, los Tribunales Constitucionales de los Länder y el resto de los tribunales ordinarios, prosiguieron utilizando la fórmula clásica». ${ }^{18}$

Paralelo a lo anterior existe un debate actual relativo a la aplicación monolítica del principio de proporcionalidad —o, cuando menos, del de arbitrariedad- en oposición a una intensidad variable a la norteamericana, modulada en relación a los requisitos del propio artículo 3.1 de la Ley Fundamental de

15 Giménez Glück, David, Op. cit., p. 91.

16 Villacorta Mancebo, Luis, «Principio de igualdad y legislador: arbitrariedad y proporcionalidad como límites (probablemente insuficientes)», en Revista de Estudios Politicos (nueva época), número 13, Madrid, 2005, p. 57.

17 Ibid., p. 58.

18 Ibid., p. 59. 
Bonn. Si bien el Tribunal Alemán ha desarrollado criterios de modulación, éstos no se encuentran definidos con la precisión norteamericana, de tal suerte que «la doctrina alemana mayoritaria insiste durante de los últimos años en la idea de encontrar "puntos de vista centrales» para determinar la intensidad del análisis por lo que hace a los tratamientos desiguales referidos tanto a las personas - categorías de personas — como al fondo del asunto». ${ }^{19}$

\section{VARIABLE INTENSIDAD EN LOS TESTS NORTEAMERICANOS EN MATERIA DE IGUALDAD}

Para nuestros efectos interesa conocer las tres categorías generales de modulación utilizadas por la Corte Suprema de los Estados Unidos como estándar en materia de igualdad, que son: rational basis review, strict scrutiny e intermediate review. Únicamente se centra en éstas porque, como se verá, se trata de categorías que sirven de referente para las jurisprudencias colombiana y mexicana.

A partir del estudio de argumentos encaminados a probar transgresiones a la decimocuarta enmienda — más conocida como equal protection clause - la Corte Suprema ha desarrollado un gran - y no siempre uniforme- acervo jurisprudencial. ${ }^{20}$ Los tests que interesan pueden explicarse de manera general en los siguientes términos:

a) Rational basis review (escrutinio racional) — Se trata del estándar más laxo de revisión y el más utilizado. El juez determina primero si la medida cuya constitucionalidad se controvierte tiene un fin razonable o si el órgano tuvo una base racional para emitirla. En caso afirmativo se determina que la medida es "legítima» y acto seguido se estudia si se encuentra razonablemente encaminada a la consecución del fin buscado. De ser así, la medida ha pasado la prueba constitucional. Es una rara ocurrencia que una política sea declarada inconstitucional bajo este escrutinio. ${ }^{21}$

19 Ibid., p. 63.

${ }^{20}$ Este trabajo presenta una visión general y conceptual de los tres tipos de tests más utilizados en la jurisprudencia norteamericana en relación con el derecho a la igualdad. Al margen de lo anterior debe notarse que el debate contemporáneo en la academia norteamericana versa sobre la aplicación de estos tests, su heterogeneidad y la creación de nuevas categorías. Para un ejemplo de estos nuevos estudios cfr. Goldberg B. Suzzane, «Equality Without Tiers», en Southern California Law Review, número 481, 2004.

${ }^{21}$ Ejemplos de casos que utilizan este tipo de escrutinio son Railway Express Agency, Inc. et al. v. New York (336 U.S. 106 (1949)) y Kotch et al. v. Board of River Port Pilot Commissioners for the Port of New Orleans (330 U.S. 552 (1947)). 
b) Strict Scrutiny (escrutinio estricto) — Se trata del estándar más exigente, el cual se utiliza cuando exista una diferenciación basada en "categorías sospechosas» como la raza o la nacionalidad. ${ }^{22}$ Ante éstas el Juez debe determinar primero si existe no una finalidad «legítima» sino un «interés vinculante y prevalente con respecto a otros» — compelling or overriding state interest-.

La racionalidad detrás de las categorías sospechosas aparece por primera vez en la famosa nota al pie del Justice Stone en el caso United States v. Carolene Products Co. (304 U.S. 144 (1983)) en cual se refiere al escrutinio estricto que debe ejercerse al examinar procesos políticos que puedan traer como resultado legislación que sea producto de prejuicios en contra de las «minorías discretas e insulares». ${ }^{23}$

${ }^{22}$ En jurisprudencia norteamericana son categorías sospechosas: raza, nacionalidad (national origin), religión e inmigración (alienage), mientras que son clasificaciones que vulneran derechos fundamentales la negación o dilución del voto, migración interestatal, acceso a la justicia y otros derechos reconocidos como fundamentales. A ambas se les aplica el test estricto.

${ }^{23}$ El famoso texto de la nota al pie número 4 establece:

There may be narrower scope for operation of the presumption of constitutionality when legislation appears on its face to be within a specific prohibition of the Constitution, such as those of the first ten amendments, which are deemed equally specific when held to be embraced within the Fourteenth. See Stromberg v. California, 283 U. S. 359, 283 U. S. 369-370; Lovell v. Griffin, 303 U. S. 444, 303 U. S. 452.

It is unnecessary to consider now whether legislation which restricts those political processes which can ordinarily be expected to bring about repeal of undesirable legislation is to be subjected to more exacting judicial scrutiny under the general prohibitions of the Fourteenth Amendment than are most other types of legislation. On restrictions upon the right to vote, see Nixon $v$. Herndon, 273 U. S. 536; Nixon v. Condon, 286 U. S. 73; on restraints upon the dissemination of information, see Near v. Minnesota ex rel. Olson, 283 U. S. 697, 283 U. S. 713-714, 283 U. S. 718-720, 283 U. S. 722; Grosjean v. American Press Co., 297 U. S. 233; Lovell v. Griffin, supra; on interferences with political organizations, see Stromberg v. California, supra, 283 U. S. 369; Fiske v. Kansas, 274 U. S. 380; Whitney v. California, 274 U. S. 357, 274 U. S. 373-378; Herndon v. Lowry, 301 U. S. 242, and see Holmes, J., in Gitlow v. New York, 268 U. S. 652, 268 U. S. 673; as to prohibition of peaceable assembly, see De Jonge v. Oregon, 299 U. S. 353, 299 U. S. 365.

Nor need we enquire whether similar considerations enter into the review of statutes directed at particular religious, Pierce v. Society of Sisters, 268 U. S. 510, or national, Meyer v. Nebraska, 262 U. S. 390; Bartels v. Iowa, 262 U. S. 404; Farrington v. Tokushige, 273 U. S. 284, or racial minorities, Nixon v. Herndon, supra; Nixon v. Condon, supra: whether prejudice against discrete and insular minorities may be a special condition, which tends seriously to curtail the operation of those political processes ordinarily to be relied upon to protect minorities, and which may call for a correspondingly more searching judicial inquiry. Compare 17 U. S. 428; South Carolina v. Barnwell Bros., @ 303 U. S. 177, 303 U. S. 184, n 2, and cases cited. (Énfasis añadido) 
Siguiendo con la aplicación del test, el fin perseguido por la medida debe ser "extremadamente importante» y no basta con una mera base racional. De satisfacerse el requisito, la medida es catalogada como "permisible» y lo siguiente es determinar si ésta es la única manera para alcanzar el fin buscado. Si hay otra manera — cualquiera - de lograrlo sin dañar a la clase sospechosa la medida será declarada inconstitucional.

El test es tan estricto que se torna virtualmente insuperable, como se ilustra en la decisión unánime de la Corte en Loving v. Virginia (388 U.S. 1 (1967)) que declara inconstitucional el Racial Integrity Act de 1924 emitido por el Estado de Virginia a fin de penalizar el matrimonio interracial. ${ }^{24}$

c) Intermediate standard of review (escrutinio intermedio) - Se trata de un punto intermedio entre los dos extremos anteriores, utilizado para las diferenciaciones con base en género - el cual si bien no tiene el grado máximo de categoría sospechosa para la jurisprudencia norteamericana, sí merece protección especial-. Bajo el presupuesto que toda medida que asume o propaga roles tradicionales de género tiene tintes de discriminatoria, en estos casos la autoridad debe mostrar un interés «importante» y que la medida esté «substancialmente relacionada» a su consecución. ${ }^{25}$

Tomando en consideración estas precisiones toca ahora entrar de lleno al estudio de las jurisprudencias colombiana y mexicana, las cuales se han caracterizado precisamente por enfocarse en el punto controvertido en Alemania: la aplicación del principio de proporcionalidad en materia de igualdad con intensidad variable.

\section{EL CASO COLOMBIANO}

El principio de proporcionalidad tiene su génesis en Colombia a través de las demandas que alegan violaciones al derecho a la igualdad y es en este campo donde ha logrado mayor desarrollo.

\footnotetext{
${ }^{24}$ Lawrence Tribe señala que es «estricto» en teoría y usualmente «fatal» en la práctica y que un caso en el cual se supere este estándar es de lo más excepcional, como sucedió en el criticado Korematsu v. United States (cfr. Tribe H., Lawrence, American Constitutional Law, $2^{a}$ Edición, Foundation Press, Nueva York, 1998, pp. 1451, 1452).

25 Casos que utilizan este escrutinio son, por ejemplo, Stanton v. Stanton (421 U.S. 7 (1975)) y Weinberger v. Wiesenfeld (420 U.S. 636 (1975)).
} 


\section{Proporcionalidad como parámetro de igualdad}

La primera vez que el principio de proporcionalidad fue utilizado en la jurisprudencia colombiana fue en la acción de tutela T-422/92 fallada el 19 de junio de 1992, en la cual se valoraba una posible violación al derecho de igualdad en relación con los requisitos para los nombramientos de servidores públicos. ${ }^{26}$

En vez de optar por la ruta tradicional para dar respuesta a las peticiones formuladas en torno al principio de igualdad, la Corte elige una solución novedosa apoyándose en la doctrina del Tribunal Europeo de Derechos Humanos. Al efecto, establece que no todo trato diferenciado traerá como consecuencia una discriminación sino sólo aquéllos que no sean justificables en términos constitucionales. Al efecto, el juez realizará un estudio atendiendo a la finalidad y los efectos de la medida controvertida, entre los cuales debe existir una relación razonable de proporcionalidad entre los medios empleados y la finalidad perseguida.

Se sostiene que el principio de proporcionalidad busca que la medida no sólo tenga un fundamento legal, sino que sea aplicada de tal manera que los intereses jurídicos de otras personas o grupos no se vean afectados, o que ello suceda en grado mínimo.

En este asunto es posible identificar el principio de un test de proporcionalidad estrictamente ligado al principio de igualdad. Debe destacarse que se trata de un esbozo primario ya que no existe todavía una conceptualización clara: primero se distingue la razonabilidad de la proporcionalidad de la norma en distintos apartados, pero posteriormente se mezclan los conceptos al hablar de la «relación razonable de proporcionalidad».

\section{Test de razonabilidad}

Dos años después aparece la figura que la Corte ha denominado «test de razonabilidad» en la acción de tutela T-230/94 fallada el 14 de mayo de 1994. En un caso que versaba sobre el derecho de igualdad de los sindicatos, se desarrolla un esquema argumentativo que sirve como plantilla para analizar el trato diferenciado a la luz de los siguientes elementos:

1. Diferencia de los supuestos de hecho.

2. Presencia de sentido normativo (fin o valor) de la diferencia de trato.

${ }^{26}$ Acción de tutela T-422/92, Jorge Eliecer Rangel Peña $v$. Recursos Naturales Renovables y del Ambiente, INDERENA, fallado por la Séptima Sala de Revisión de la Corte Constitucional, 1992. 
3. Razonabilidad - Validez constitucional del sentido (fin) propuesto.

4. Racionalidad - Eficacia en la relación entre hechos, norma y fin.

5. Adecuación - Proporcionalidad entre medios y fines.

La Corte resalta la labor particular que debe llevar a cabo el juez en este test, pues no se trata de una adecuación lógica de lo particular a lo general sino de comprender la relación de adecuación entre los elementos indicados. Es una labor argumentativa en la que los elementos forman un todo dotado de sentido y no simplemente una sumatoria de partes separables.

En la jurisprudencia colombiana la lógica predominante en el examen de la igualdad es aquella de la razonabilidad, fundada en la ponderación y sopesación de valores y no simplemente en la confrontación lógica de los mismos.

En esta línea de razonamiento se delimita el «test de igualdad» — término que parece utilizado como una subespecie del test de razonabilidad - de la siguiente manera: el trato diferenciado de dos situaciones de hecho diversas no constituye una discriminación siempre y cuando se cumplan las siguientes condiciones: a) que los hechos sean distintos; b) que la decisión de tratarlos de manera diferente esté fundada en un fin aceptado constitucionalmente; c) que la consecución de dicho fin por los medios propuestos sea posible y además adecuada.

La Corte Colombiana se tomó la aplicación del «test de razonabilidad» en serio, incluyendo en el texto de la sentencia argumentos encaminados a demostrar que se trata de un mecanismo idóneo para lidiar con la adjudicación de derechos constitucionales y no un simple cambio de criterio caprichoso. En suma, no se está ante un caso en el cual se importa un modelo de la doctrina internacional sin mayor justificación sino que la Corte entiende la relevancia del cambio de criterio y los efectos que ello conlleva, pero decantarse por este nuevo mecanismo le parece razonable en aras de los beneficios que trae consigo. Al efecto, establece:

2.3. El carácter normativo de todos los enunciados constitucionales y el principio de la efectividad de los derechos, imponen, hoy más que nunca, este tipo de razonamiento. Si la Carta de derechos demanda del juez constitucional decisiones en derecho que sean a la vez justas, en las cuales los valores y principios esenciales del ordenamiento encuentren realización, forzoso es entonces concluir que los procedimientos formales de validez no siempre serán el camino para el descubrimiento de la solución ideal. Dicho en otros términos, la primacía constitucional de los postulados axiológicos fundamentales, condiciona el seguimiento de los criterios positivos de validez normativa al respeto de los valores y principios. $^{27}$

27 Acción de tutela T-230/94, Juan de Jesús Jiménez v. COOP-FEBOR, emitido por la Tercera Sala de Revisión de la Corte Constitucional, 1994. 
La versión finalizada de esta metodología se encuentra en la demanda de constitucionalidad C-022/96 — un caso que trataba de los privilegios otorgados a los estudiantes que habían completado el servicio militar- fallado el 23 de enero de $1996 .^{28}$ En esta ocasión la Corte dedica un análisis más detallado a los elementos de la proporcionalidad — tal y como son aplicados por el Tribunal alemán- dentro de su propio test de razonabilidad.

En cuanto al principio de proporcionalidad se refiere, la Corte explica que en la teoría jurídica alemana se ha mostrado cómo el concepto de razonabilidad puede ser aplicado satisfactoriamente sólo si se concreta en otro más específico, el de proporcionalidad. Señala que éste sirve como punto de apoyo de la ponderación entre principios constitucionales: cuando dos principios entran en colisión, porque la aplicación de uno implica la reducción del campo de aplicación de otro, corresponde al juez constitucional determinar si esa reducción es proporcionada a la luz de la importancia del principio afectado.

Continúa explicando que el concepto de proporcionalidad comprende tres conceptos parciales: la adecuación de los medios escogidos para la consecución del fin perseguido; la necesidad de la utilización de esos medios para el logro del fin - esto es, que no exista otro medio que pueda conducir al fin y que sacrifique en menor medida los principios constitucionales afectados por el uso de esos medios - y la proporcionalidad en sentido estricto entre medios y fin, es decir, que el principio satisfecho por el logro de este fin no sacrifique principios constitucionalmente más importantes.

Posteriormente aterriza el concepto de proporcionalidad al principio de igualdad, explicando que en el análisis de tratos diferenciados un trato desigual no vulnera ese principio sólo si se demuestra que es (1) adecuado para el logro de un fin constitucionalmente válido; (2) necesario, es decir, que no existe un medio menos oneroso, en términos del sacrificio de otros principios constitucionales, para alcanzar el fin; y (3) proporcionado, esto es, que el trato desigual no sacrifica valores y principios - dentro de los cuales se encuentra el principio de igualdad- que tengan un mayor peso que el principio que se quiere satisfacer mediante dicho trato.

El test completo —identificado como una guía metodológica- queda articulado en esta sentencia. Consiste de tres pasos que comprenden el «test de razonabilidad» en sí, dentro del cual el tercero de sus elementos se desdobla en tres más — tal y como lo hace el Tribunal alemán y acorde a lo señalado por Alexy-. Los pasos a seguir son:

${ }^{28}$ Demanda de inconstitucionalidad C-022/1996 enderezada por Álvaro Montenegro García, fallada por la Corte Constitucional Colombiana en Pleno, 1996. 

igual.

$1^{\circ}$ Identificar el objetivo que persigue la medida que implica el trato des-

$2^{\circ}$ Verificar que la finalidad sea constitucionalmente admisible.

$3^{\circ}$ Evaluar la razonabilidad del trato al sopesar la relación entre los medios y fines, siguiendo los siguientes subpasos:

a) Adecuación - Que los medios escogidos sean adecuados para lograr el fin perseguido.

b) Indispensabilidad - La necesidad de la utilización de los medios para el logro del fin.

c) Proporcionalidad en sentido estricto - Se traduce en que la medida no sacrifique valores y principios - dentro de los cuales se encuentra el principio de igualdad- que tengan un mayor peso que el principio que se busca satisfacer.

Debe notarse el uso heterogéneo de los términos "proporcionalidad en sentido estricto" y "principio proporcionalidad». Al enunciar el test se habla de lo primero como de una cuestión cuantitativa: que el principio que se busca satisfacer no sacrifique otros principios de mayor cuantía. Posteriormente la Corte alude a su propia jurisprudencia acerca del «principio de proporcionalidad» estableciendo que la medida debe aplicarse de tal manera que no afecte los intereses de otras personas o que ello suceda en la menor medida posible.

Claramente estos dos conceptos no se identifican con la terminología alemana. Lo que se verá en los apartados siguientes es mayor atención a detalle en el desarrollo jurisprudencial y el subsiguiente perfeccionamiento del modelo con la aplicación de la proporcionalidad en sentido estricto alemana sólo cuando se trate de casos que exijan un escrutinio reforzado.

\section{Tres tipos de tests}

La adecuación del test europeo a la justicia colombiana no ha sido el único logro de esta Corte, sino que en su jurisprudencia se distinguen otros dos modelos diferenciados. Carlos Bernal Pulido explica que es posible distinguir tres diferentes tipos de tests: uno europeo basado en la proporcionalidad con intensidad constante, otro americano que se matiza con base en distintos niveles de intensidad y un tercero que combina los dos anteriores. Los tres modelos son:

a) El juicio de igualdad como principio de proporcionalidad en la jurisprudencia de la Corte Constitucional. Se trata del primer modelo utilizado, que 
adopta los elementos básicos del juicio de igualdad que es aplicado por el Tribunal Europeo de Derechos Humanos, el Tribunal Constitucional Español y el Tribunal Constitucional Alemán —este último a partir de la «nueva fórmula»— y que se estructura con base en el principio de proporcionalidad. La Corte se refiere a este juicio indistintamente como de "proporcionalidad» o de «razonabilidad», aunque en sentido estricto estos términos no son sinónimos.

b) El juicio de igualdad con tres tipos de escrutinios. El segundo modelo utilizado está basado en la jurisprudencia de la Corte Suprema de Estados Unidos sobre la aplicación de la cláusula de la equal protection prevista por la decimocuarta enmienda. Se utilizan distintos niveles de intensidad en los «escrutinios» $\mathrm{o}$ «tests» de igualdad, sean estrictos, intermedios o débiles.

c) El juicio integrado de la igualdad. En la Sentencia C-93 de 2001 la Corte Constitucional intentó construir un «juicio integrado de igualdad», que combinara las ventajas del juicio de igualdad de índole europea - estructurado alrededor del principio de proporcionalidad - con las ventajas del juicio norteamericano. Con el uso de este método se aprovecha tanto la mayor claridad analítica que ofrece el procedimiento de la proporcionalidad, como la posibilidad de que cada uno de sus subprincipios sea aplicado con una intensidad diferente, de acuerdo con la extensión del ámbito de apreciación que el Legislador o la Administración tenga en la materia que sea relevante. ${ }^{29}$

Los siguientes apartados se dedicarán al estudio del tercer tipo de test, el cual se considera un ejemplo paradigmático de tropicalización.

\section{Test(s) integrado(s)}

Es particularmente relevante para este estudio el análisis del tercer tipo de test — denominado «juicio integrado de igualdad»— dado que no sólo constituye un ejemplo de tropicalización en términos de la hipótesis que sostiene este trabajo sino porque, como se verá, sirve de inspiración para el modelo utilizado por la Corte mexicana.

Si bien tal y como apunta Bernal Pulido este test integrado aparece en el año 2001, conviene analizar su versión más perfeccionada y reciente visible en la cuestión de inconstitucionalidad C-227/2004 — que versa sobre las pensiones de

${ }^{29}$ Cfr. Bernal Pulido, Carlos, El juicio de la igualdad en la jurisprudencia de la Corte Constitucional Colombiana, Universidad Externado de Colombia, Colombia, pp. 5, 8, 13, disponible en: http://www.cajpe.org.pe/rij/bases/nodiscriminacion/BERNAL.PDF. 
invalidez para hijos menores de 18 años—. ${ }^{30}$ Se trata no de un solo test sino de tres tipos con características particulares y exigencias variadas.

En primer término la sentencia establece que la importancia del juicio o test de igualdad radica en que intenta dar objetividad y previsibilidad a los exámenes de igualdad que realizan los tribunales. Ello permite, a su vez, que la ciudadanía pueda hacer un mejor seguimiento y un escrutinio más exacto de las decisiones de los jueces, tal como cabe hacerlo en relación con todas las autoridades públicas. Además, ofrece guías claras al legislador al momento de diseñar normas que establecen distinciones entre grupos de personas.

En segundo término la jurisprudencia sostiene que el control de constitucionalidad en general y el juicio de igualdad en particular adoptan diversas modalidades - leve, intermedio o estricto — según su grado de intensidad. La intensidad del control de constitucionalidad y del juicio de igualdad varía dependiendo de la materia objeto de la norma demandada y sus implicaciones, si bien en todo caso es necesario examinar las circunstancias concretas que configuran cada situación para determinar el nivel de intensidad del juicio al que ha de ser sometida una norma que es objeto de control de constitucionalidad.

De esta manera, se trata de un desarrollo más complejo de tres tipos de tests que atienden a los tres grados de intensidad señalados en la jurisprudencia norteamericana. Al preguntarse por la relación entre los medios y fines de la medida la Corte atiende al ámbito material y, con base en ello, elige una plantilla de entre los siguientes modelos:

a) Juicio leve . El medio debe ser adecuado para conseguir el fin legítimo y ninguno de ellos puede estar constitucionalmente prohibido. De cumplirse con estos requisitos, ambos son denominados «legítimos».

Materialmente se aplica en lo económico, tributario, política internacional, competencias específicas de órganos constitucionales, normas preconstitucionales derogadas - pero que mantengan efectos - y cuando del contexto del artículo demandado no se advierten indicios prima facie de amenazas para el derecho cuestionado.

b) Juicio intermedio. El medio debe ser adecuado y efectivamente conducente para alcanzar el fin buscado. Éste debe ser no sólo legítimo sino importante al promover intereses públicos reconocidos constitucionalmente o responder a problemas importantes que exijan respuestas del Estado.

${ }^{30}$ Cuestión de constitucionalidad C-227/2004 enderezada por Nelson Ruiz Velásquez y fallada por el Pleno de la Corte Constitucional de Colombia, 2004. 
Materialmente se aplica sobre derechos constitucionales no fundamentales o cuando exista un indicio de arbitrariedad relejado en la afectación grave de la libre competencia.

c) Juicio estricto. El medio debe ser adecuado, efectivamente conducente y necesario — que no pueda reemplazarse por otro menos dañino- para alcanzar el fin. Éste debe ser legítimo, importante y además imperioso. Es el único de los tests que, aunado a los requisitos anteriores, se pregunta por la proporcionalidad en sentido estricto: los beneficios de adoptar la medida deben exceder claramente las restricciones que ella implica sobre otros principios y valores constitucionales.

Materialmente se aplica sobre: 1) categorías sospechosas vedadas por el inciso $1^{\circ}$ del artículo 13 de la Constitución; 2) afectación a personas en condiciones de debilidad manifiesta, grupos marginados o discriminados, sectores sin acceso efectivo a la toma de decisiones o - utilizando la traducción literal de la terminología norteamericana- «minorías insulares y discretas»; 3) cuando aparece prima facie que la medida diferenciadora afecta gravemente el goce de un derecho constitucional fundamental; y 4) cuando la medida crea privilegios.

Parece que en estos momentos la Corte colombiana ha abandonado ya la rigidez del test tripartito alemán para crear un modelo mucho más flexible que distingue tres tests particulares cuyas categorías son de absoluta inspiración norteamericana, lo cual se evidencia por la utilización de términos como «fin imperioso", "categorías sospechosas» y «minorías insulares y discretas».

Los requisitos no son diferentes sino acumulativos. Esto es, entre más se ascienda en la escala de importancia constitucional, se tendrán que satisfacer los requisitos del nivel anterior aunadas a las del siguiente peldaño.

En suma, es razonable afirmar que la Corte colombiana ha desarrollado un entramado jurisprudencial complejo conformado en parte por la aplicación de los tests europeos y norteamericanos a sus propias circunstancias y, en otra, mediante la tropicalización visible en la creación de nueva doctrina que combina tanto elementos de los paradigmas originales como novedosas características surgidas a partir de sus circunstancias particulares y que constituye una herramienta fundamental en su análisis constitucional.

\section{EL CASO MEXICANO}

A pesar de que la Corte Mexicana es más antigua que su contraparte colombiana, su historia en relación al principio de proporcionalidad es muy reciente. Fue en 2004 cuando comenzó a utilizar esta figura, por medio de lo que también denomina "test de razonabilidad» — aplicado de manera destacada en el 
campo de igualdad - basado en el test colombiano integrado pero con sus propias particularidades para la modulación de intensidad.

\section{Surgimiento del «test de razonabilidad»}

La versión Mexicana del «test de razonabilidad» surge en los amparos ADR 988/2004 y AR 1629/2004. El primero es un caso de derecho penal y el segundo de materia fiscal, pero ambos surgen a partir de demandas que versan sobre el derecho a la igualdad. Son los primeros precedentes visibles en la tesis de rubro «IGUALDAD. CRITERIOS PARA DETERMINAR SI EL LEGISLADOR RESPETA ESE PRINCIPIO CONSTITUCIONAL» en la cual se asienta el test. ${ }^{31}$

En esta jurisprudencia se explica que el principio de igualdad debe entenderse como la exigencia constitucional de tratar igual a los iguales y desigual a los desiguales y de ahí que en algunas ocasiones hacer distinciones estará vedado, mientras que en otras estará permitido o incluso constitucionalmente exigido.

En ese tenor cuando la Corte conoce de un caso en el cual la ley distingue entre dos o varios hechos, sucesos, personas o colectivos, debe analizar si dicha distinción descansa en una base objetiva y razonable o si, por el contrario, constituye una discriminación constitucionalmente vedada.

En los términos de la jurisprudencia citada esta Corte establece su propio test de razonabilidad que — en materia de igualdad — será aplicado de la siguiente manera:

a) Primero, determinar si el fin es objetivo y admisible en términos constitucionales: el legislador no puede introducir tratos desiguales de manera arbitraria, sino que debe hacerlo con el fin de avanzar en la consecución de objetivos admisibles dentro de los límites marcados por las previsiones constitucionales o expresamente incluidos en ellas.

b) Segundo, analizar la racionalidad de la medida, lo cual se traduce en una relación instrumental entre medios y fines: es necesario que la introducción de una distinción constituya un medio apto para conducir al fin u objetivo que el legislador quiere alcanzar, es decir, que exista una relación de instrumentalidad entre la medida clasificatoria y el fin pretendido

31 Tesis de jurisprudencia 1a./J. 55/2006, Primera Sala de la Suprema Corte de Justicia de la Nación (Semanario Judicial de la Federación y su Gaceta, Novena Época, tomo XXIV, Septiembre de 2006, 75). 
c) Tercero, estudiar la proporcionalidad entre medios y fines: a efecto de determinar si la búsqueda de una finalidad constitucionalmente válida no produce una trasgresión innecesaria o excesiva de otros valores constitucionales, verificando si hay una medida que conlleve claramente menores afectaciones y que consiga igualmente el fin buscado.

En el requisito de proporcionalidad la Corte argumenta que el legislador no puede tratar de alcanzar objetivos constitucionalmente legítimos de un modo abiertamente desproporcional, de manera que el juzgadora ha de determinar si la distinción legislativa se encuentra dentro del abanico de tratamientos que satisfagan este requisito, tomando en cuenta la situación de hecho, la finalidad de la ley y los bienes y derechos constitucionales afectados por ella.

Por último la jurisprudencia razona que es de gran importancia determinar en cada caso respecto de qué se está predicando la igualdad, porque esta última constituye un principio y un derecho de carácter fundamentalmente adjetivo que se predica siempre de algo y se trata de un referente relevante al momento de realizar el control de constitucionalidad de las leyes.

En esta línea argumentativa se enfatiza la necesidad de una intensidad variable en razón de que el propio texto constitucional mexicano permite que en algunos ámbitos el legislador tenga mayor amplitud para desarrollar su labor normativa, mientras que en otros insta al Juez a ser especialmente exigente cuando deba determinar si aquél ha respetado las exigencias derivadas del principio mencionado.

Habiendo estudiado la jurisprudencia colombiana puede llegarse a la conclusión de que el test utilizado en México es casi idéntico al test integrado, incluso hasta en la terminología utilizada. Con todo, es importante distinguir una diferencia relevante: la variación Mexicana nunca llega al máximo nivel de la proporcionalidad en sentido estricto — como se dijo, núcleo del test alemán— lo cual trae como consecuencia lógica que se declare la inconstitucionalidad de muchos menos actos en comparación a lo que sucedería si se utilizara el estándar más exigente que constituye la proporcionalidad en sentido estricto.

\section{Modulación en el escrutinio mexicano}

Añadiendo su propio toque de tropicalización la Corte mexicana emitió otra jurisprudencia titulada «IGUALDAD. CASOS EN LOS QUE EL JUEZ CONSTITUCIONAL DEBE HACER UN ESCRUTINIO ESTRICTO DE LAS CLASIFICACIONES LEGISLATIVAS (INTERPRETACIÓN DEL AR- 
TÍCULO 1o. DE LA CONSTITUCIÓN POLÍTICA DE LOS ESTADOS UNIDOS MEXICANOS)» con el ánimo de establecer grados diferenciados de intensidad en el escrutinio en la línea del test integrado de Colombia, pero a la luz de las particularidades del Artículo $1^{\circ}$ de la Constitución Política de los Estados Unidos Mexicanos. ${ }^{32}$

Realiza la modulación con base en el artículo 1o. para definir los casos en los que procede escrutinio estricto, razonando que a partir de su texto se manifiesta la voluntad constitucional de asegurar en los más amplios términos el goce de los derechos fundamentales y de que las limitaciones a ellos sean concebidas restrictivamente, de conformidad con el carácter excepcional que la Constitución les atribuye.

En este orden de ideas se establece que siempre que la acción clasificadora del legislador incida en los derechos fundamentales garantizados constitucionalmente, será necesario aplicar con especial intensidad las exigencias derivadas del principio de igualdad y no discriminación.

Para esta Corte la intención constitucional es extender las garantías implícitas en el principio de igualdad al ámbito de las acciones legislativas que tienen un impacto significativo en la libertad y la dignidad de las personas, así como al de aquellas que se articulan en torno al uso de una serie de "criterios clasificatorios» mencionados en el tercer párrafo del artículo $1^{\circ}$. Aclara que ello no implica que al legislador le esté vedado absolutamente el uso de dichas categorías en el desarrollo de su labor normativa, sino que debe ser especialmente cuidadoso al hacerlo.

Las categorías en cuestión son origen étnico o nacional, género, edad, capacidades diferentes, condición social, condiciones de salud, religión, opiniones, preferencias, estado civil o en cualquier otro que atente contra la dignidad humana y tenga por objeto anular o menoscabar los derechos y libertades de las personas.

En los términos de la citada jurisprudencia, la interpretación del artículo $1^{\circ}$ de la Constitución Mexicana permitió que la Corte construyera un estándar para su propio strict scrutiny — llamando a un análisis «especialmente cuidadoso»- al aplicar el test de razonabilidad. Como se dijo, debe notarse que si bien en este escrutinio se exige mayor cuidado no se trata de un análisis fuerte con proporcionalidad en sentido estricto como se hace en la jurisprudencia colombiana.

Posteriormente también desarrolló un estándar para un análisis más débil — cercano al norteamericano rational basis review - en el campo económico,

32 Tesis de jurisprudencia 1a./J. 37/2008, Primera Sala de la Suprema Corte de Justicia de la Nación (Semanario Judicial de la Federación y su Gaceta, Novena Época, tomo XXVII, abril de 2008, 175). 
mediante la jurisprudencia de rubro «ANÁLISIS CONSTITUCIONAL. SU INTENSIDAD A LA LUZ DE LOS PRINCIPIOS DEMOCRÁTICO Y DE DIVISIÓN DE PODERES». ${ }^{33}$

En esta jurisprudencia se sostiene que la fuerza normativa del principio democrático y del principio de separación de poderes tiene como consecuencia obvia que los otros órganos del Estado — y entre ellos, el juzgador constitucionaldeben respetar la libertad de configuración con que cuentan el Congreso y el Ejecutivo en el marco de sus atribuciones.

Así, apunta que la severidad del control judicial se encuentra inversamente relacionada con el grado de libertad de configuración por parte de los autores de la norma. Considera evidente que la Constitución Federal exige una modulación del juicio de igualdad sin que eso implique ninguna renuncia de la Corte al estricto ejercicio de sus competencias de control.

Para esta Corte el caso de normatividad con efectos económicos o tributarios exige que, por regla general, la intensidad del análisis constitucional deba ser poco estricta con el fin de no vulnerar la libertad política del legislador en campos como el económico.

En este orden de ideas razona que la propia Constitución establece una amplia capacidad de intervención y regulación diferenciada del Estado, considerando que cuando el texto constitucional establece un margen de discrecionalidad en ciertas materias, ello significa que las posibilidades de injerencia del Juez constitucional son menores y, por ende, la intensidad de su control se ve limitada.

Utilizando un argumento de división de poderes, establece que en tales esferas un control muy estricto llevaría al Juez constitucional a sustituir la competencia legislativa del Congreso -o la extraordinaria que puede corresponder al Ejecutivo- pues no es función del Poder Judicial Federal sino de los órganos políticos entrar a analizar si esas clasificaciones económicas son las mejores o resultan necesarias.

A partir del entramado formado por las jurisprudencias citadas la Corte Mexicana ha creado su propio esquema de proporcionalidad utilizando una mezcla del test integrado colombiano y sus propios mandatos constitucionales, si bien no se aprecia el grado de precisión alcanzado en Colombia.

33 ANÁLISIS CONSTITUCIONAL. SU INTENSIDAD A LA LUZ DE LOS PRINCIPIOS DEMOCRÁTICO Y DE DIVISIÓN DE PODERES. Tesis de jurisprudencia 1a./J. 84/2006, Primera Sala de la Suprema Corte de Justicia de la Nación (Semanario Judicial de la Federación y su Gaceta, tomo XXIV, Noviembre de 2006, 29). 


\section{Aplicación finalizada del test mexicano}

El panorama completo puede ser explicado en los siguientes términos: con base en los mandatos contenidos en la propia Constitución la severidad del control judicial se encuentra inversamente relacionada con el grado de libertad de configuración por parte de los autores de la norma.

En un extremo se encuentra el strict scrutiny — denominado «análisis de especial intensidad»— en los casos en los que la norma tildada de inconstitucional incida en los derechos fundamentales garantizados constitucionalmente; mientras que en el otro se sitúa el rational basis review — que la Corte llama de intensidad "poco estricta» - el cual es utilizado en materia económica. La constante en esta ecuación es la aplicación del test de razonabilidad en sí mismo mientras que la variable es la intensidad del análisis atendiendo a los criterios apuntados.

A diferencia de la metodología europea aquí el pronunciamiento sobre la intensidad del análisis forma parte del proceso argumentativo. Para la materia de igualdad este proceso puede explicarse atendiendo a la siguiente serie de preguntas:

1. ¿Existe una argumentación por parte del actor en el juicio constitucional?

2. ¿Ha aportado el actor el tertium comparationis que supone el parámetro para realizar el análisis de igualdad y la contraparte soportado la carga argumentativa de la prueba?

3. ¿Se encuentra el tertium comparationis dentro del ámbito de aplicación de la garantía de igualdad?

4. ¿El trato diferenciado se realiza con base en categorías sospechosas? Si la respuesta es positiva lo suyo es proceder a la aplicación del test de razonabilidad en modalidad de strict scrutiny.

5. De no ser así, entonces ¿cuál es la intensidad requerida? Queda claro que si se trata de materia económica la jurisprudencia apunta hacia el rational basis review, pero debe destacarse que no se han emitido criterios que establezcan una intensidad intermedia entre los dos extremos, esto es, un modelo parecido al intermediate scrutiny.

6. ¿Es justificado el trato diferenciado? Finamente en este punto se procede a la aplicación del «test de razonabilidad» en sí.

Para ejemplificar este modelo argumentativo utilizando casos materialmente claros: por un lado, en un asunto de tipo penal que infrinja en el derecho fundamental a la libertad personal por imponer pena de cárcel el juez debe pre- 
guntarse si el camino escogido por el legislador era el más adecuado para lograr el objetivo deseado. Por el otro, en un caso fiscal la Corte debe restringir la intensidad del análisis y solamente preguntarse si no existe una trasgresión innecesaria o "grosera» a otros derechos o valores constitucionales, pero no invalidará por advertir otra política fiscal que estime más adecuada, debido a que hacerlo conllevaría una violación al principio democrático y a la división de poderes.

Toda vez que la mayoría de la carga de trabajo soportada por la Corte Mexicana es formada por demandas fiscales, los más de los asuntos serán analizados bajo rational basis review que tradicionalmente constituye un obstáculo de fácil superación para el legislador diligente que mínimamente se haya ocupado de cumplir con el requisito de motivación legislativa.

Es importante notar que a diferencia de la vía tomada por la Corte Colombiana, la Suprema Corte Mexicana no realiza referencia alguna ni a la jurisprudencia de las cortes europeas ni a la de aquélla, pese a que parece ser la influencia más directa en el desarrollo de estos nuevos alcances.

\section{CONCLUSIÓN: LA TROPICALIZACIÓN DEL PRINCIPIO DE PROPORCIONALIDAD}

Tras este recorrido por las jurisprudencias que las Cortes Colombiana y Mexicana han desarrollado a partir de los principios de proporcionalidad e igualdad, la inevitable conclusión es la confirmación de la hipótesis inicial: que éstas han tropicalizado el juicio europeo mediante una combinación de los tests norteamericanos y el uso de las particularidades de cada jurisdicción. Adicionalmente pueden realizarse otras observaciones:

Relativo a Colombia, debe decirse que esta Corte tiene una importante ventaja respecto de la Mexicana en cuanto a la temporalidad de la incorporación del principio de proporcionalidad, lo cual conlleva una mayor sofisticación y perfeccionamiento en cuanto a su aplicación.

Debido a ello la jurisprudencia de Colombia cuenta con una variedad de pronunciamientos y una línea - en términos generales - clara en su desarrollo a lo largo de más de una década. Se estima que son valiosas las referencias expresas al derecho comparado toda vez que le dan un mayor grado de legitimación a la decisión de importar un modelo que se ha sido probado exitosamente en otras Cortes que gozan de gran prestigio y cuyos resultados han sido positivos.

En cuanto a México, se considera que una vez que la Corte ha tomado la decisión de adoptar el «test de razonabilidad» ahora tiene el deber de continuar su 
desarrollo y perfeccionamiento, atendiendo a los valores de seguridad jurídica e igualdad en la aplicación de la ley.

Por lo que hace a la decisión de abstenerse de adoptar la proporcionalidad en sentido estricto, se estima que es un aspecto que debe ser reconsiderado en aras de que su uso trae como beneficio una mayor sujeción de las autoridades al texto constitucional.

En el estado actual de cosas en México, los productos finalizados resultan más cercanos a los modelos utilizados en Norteamérica en cuanto al rational basis review, por un lado, y el strict scrutiny en combinación con una versión menos exigente del test europeo, por el otro.

No puede dejar de mencionarse que estas experiencias de sincretismo bien podrían ser vistas como mejoras en los modelos que les dieron origen, en cuanto a la posibilidad real de aplicar el principio de proporcionalidad al de igualdad con la intensidad variable que éste exige.

Como última reflexión de este ejercicio, conviene subrayarse una preocupación que subyace al tema central del mismo: la necesaria igualdad en la aplicación de criterios por parte de los órganos jurisdiccionales. Éste es uno de los ideales que se encuentran reflejados en la doctrina norteamericana del stare decisis —obligatoriedad del precedente- que se consagra como uno de los principios fundamentales que constriñe el actuar jurisdiccional. En el ámbito europeo se habla de la igualdad en la aplicación de la ley por parte de los órganos jurisdiccionales y es una cuestión que ha ocupado a la jurisprudencia constitucional de, entre otros, el Tribunal Constitucional Español. ${ }^{34}$

El estudio realizado respecto de las decisiones de las cortes latinoamericanas ofrece una oportunidad para reflexionar en abstracto sobre este punto. La aplicación de doctrinas novedosas es a todas luces un fin legítimo pero debe realizarse de manera reflexiva. Una vez que se ha decretado la utilización de una herramienta argumentativa como es el principio de proporcionalidad en el ámbito de la igualdad, existe la obligación de continuar empleándolo para casos similares de manera constante y uniforme.

No se trata únicamente de la necesidad de coherencia sistemática ad intra, sino de una exigencia derivada de toda Constitución que consagre el principio de seguridad jurídica. El Estado de derecho efectivamente deja de existir si un órgano estatal puede situarse por encima del derecho establecido y los tribunales constitucionales no son la excepción a esta máxima. Si bien la tarea del juez no se limita a una simple aplicación de la ley, su competencia sólo le permite

34 Ver, entre otras, la doctrina fijada por la STC 8/81, seguida por los casos SSTC 49/82, 66/87, 144/88, 112/96, 2/97, 2/98 y 150/01. 
desplazarse dentro del marco del derecho y no sustituirlo arbitrariamente por sus propias concepciones.

A efecto de no caer en una "dictadura de togas", debe reconocerse que para resolver una controversia se cuenta con arbitrio mas no con arbitrariedad. Tal y como señala la propia Corte Colombiana «la igualdad en la aplicación de la ley está íntimamente ligada a la seguridad jurídica que descansa en la existencia de un ordenamiento universal y objetivo, que con idéntica intensidad obliga a todos, autoridades y ciudadanos».35

Sujetar a los tribunales a la adhesión a sus propios criterios no implica anular el arbitrio jurisdiccional. Un tribunal siempre podrá reconsiderar que un nuevo caso tiene características relevantes diferentes a las del anterior y que ello amerita un cambio en el sentido del fallo, pero siempre debe dar razones para justificar su proceder.

$\mathrm{Al}$ ser el sistema jurídico un continuo dinámico de posibilidades, siempre existirá la ocasión para volver sobre un tema que se había dado por zanjado y preguntarse si las circunstancias han permanecido constantes o si ha llegado el momento de modificar el criterio. De igual manera cabe la posibilidad de aceptar que el modelo argumentativo elegido - como sería el principio de proporcionalidad - no trajo los beneficios esperados y que éste debe abandonarse, dando argumentos para que la decisión se considere aceptable.

En última instancia se invita al lector a reflexionar sobre las palabras de Jeremy Bentham: «las buenas decisiones son aquéllas respecto de las cuales pueden aducirse buenas razones». ${ }^{36}$

Title

THE TROPICALIZATION OF PROPORTIONALITY BALANCING: THE COLOMBIAN AND MEXICAN EXPERIENCE IN EQUAL PROTECTION CLAIMS

\section{Resumen}

En «La tropicalización del principio de proporcionalidad: la experiencia de Colombia y México en el ámbito de igualdad» el autor

35 Extracto de la acción de tutela promovida por Seguros Alfa S.A., radicada con el número T-231-94, emitida por la Sala Tercera de Revisión de la Corte Constitucional el 13 de mayo de 1994.

36 Bentham, Jeremy, «Rationale of Judicial Evidence, Specially Applied to English Practice», en The Works of Jeremy Bentham, tomo VI, Bowring, Nueva York, 1962, p. 365. 
analiza cómo el principio de proporcionalidad de origen alemán fue exportado a América Latina, por medio del estudio de la jurisprudencia de la Corte Constitucional Colombiana y la Suprema Corte de Justicia Mexicana.

Toda vez que los países de esta región tradicionalmente tienden a ser más receptivos de las modas norteamericanas en oposición a las europeas, este trabajo trata de analizar cómo Colombia y México han importado el referido principio, preguntándose si han adoptado el modelo tradicional alemán, si han retenido la acostumbrada influencia norteamericana y si han aportado nuevos elementos que, incluso, pudieran ser mejoras para los modelos que les dieron origen.

El presente trabajo pretende brindar un análisis práctico de la jurisprudencia producida por las Cortes colombiana y mexicana, exponiendo primero un resumen conceptual tanto del principio de proporcionalidad en el sentido alemán como de la jurisprudencia Americana de igualdad consagrada en la Decimocuarta enmienda para posteriormente identificar estos elementos en las sentencias latinoamericanas, en conjunto con las particularidades creadas por cada una de las Cortes.

El análisis es guiado por las siguientes preguntas: ¿qué es el principio de proporcionalidad? ¿Cuál es la relación entre los principios de proporcionalidad e igualdad? ¿Cómo se han utilizado por las cortes colombiana y mexicana y cuáles son sus influencias? ¿Se hacen referencias expresas al derecho comparado para justificar la inclusión del test? ¿Han importado el tradicional test Europeo? ¿Se ha retenido la influencia norteamericana?

En última instancia se concluye que en el proceso de adaptación del principio de proporcionalidad a las circunstancias de sus propias jurisdicciones, ambas cortes han tropicalizado el modelo. Este término no es en absoluto utilizado en afán peyorativo sino como una manera de identificar y explicar una parte del proceso globalizador visible en la dinámica de las diversas cortes constitucionales, en virtud del cual las doctrinas son importadas y adaptadas a las necesidades de cada país. Idealmente el producto final aporta los beneficios disfrutados en otras jurisdicciones sin dejar de lado las particularidades del Estado receptor.

\begin{abstract}
In «The Tropicalization of Proportionality Balancing: the Colombian and Mexican Experience in Equal Protection Claims» the author analyzes how the German based proportionality balancing test was exported to Latin America, by studying the Colombian Constitutional Court and the Mexican Supreme Court.
\end{abstract}


Even though traditionally countries in this region have been influenced by American trends, this work deals with how proportionality has infiltrated Colombia and Mexico, aimed at identifying if the countries have imported the traditional European model, or if they have retained American influence.

This article is meant to provide a practical analysis of the jurisprudence produced by both Courts first giving the reader a conceptual overview of both proportionality balancing as applied by the German Court and American jurisprudence related to the equal protection clause established in the 14th Amendment. These elements are then identified in the Latin American jurisprudence, in conjunction to the particular traits created by each Court.

This work is guided by the following questions: what is proportionality balancing? How are proportionality and the principle of equality related? How has it been used by the Colombian and Mexican jurisprudences and what are its influences? Do the Courts cite other jurisdictions when using the test? Have they imported a traditional European test? Is there a visible North American influence?

The study of the Latin American examples leads to the conclusion that the Courts have «tropicalized" proportionality balancing. In this context, the term "tropicalization" is used to describe the fact that the Courts have made the test their own, adjusting it to their particular jurisprudence by combining elements from the original German test, the American based differentiated levels of scrutiny, and elements from their own constitutional standards.

Palabras clave:

Proporcionalidad, Colombia, México, Igualdad.

\section{Key Words}

Proportionality, Colombia, Mexico, Equality. 